\title{
Functional and Mechanistic Interplay of Host and Viral Alternative Splicing Regulation during Influenza Infection
}

\author{
Matthew G. Thompson and KRISTEN W. Lynch \\ Department of Biochemistry and Biophysics Perelman School of Medicine, University \\ of Pennsylvania, Philadelphia, Pennsylvania 19104, USA \\ Correspondence: klync@pennmedicine.upenn.edu
}

\begin{abstract}
Alternative splicing is a pervasive gene regulatory mechanism utilized by both mammalian cells and viruses to expand their genomic coding capacity. The process of splicing and the RNA sequences that guide this process are the same in mammalian and viral transcripts; however, viruses lack the splicing machinery and therefore must usurp both the host spliceosome and many of the associated regulatory proteins in order to correctly process their genes. Here, we use the example of the influenza Avirus to both describe how viruses utilize host splicing factors to regulate their own splicing and provide examples of how viral infection can, in turn, alter host splicing. Importantly, we show that at least some of the viral-induced changes in host splicing occur in genes that alter the efficiency of influenza replication. We emphasize the importance of increased understanding of the mechanistic interplay between host and viral splicing, and its functional consequences, in uncovering potential antiviral vulnerabilities.
\end{abstract}

Influenza A virus (IAV) is a ubiquitous and significant health threat, resulting in 290,000-650,000 deaths per year worldwide (World Health Organization 2019). In the United States alone, IAV is estimated to result $12,000-56,000$ deaths annually (Centers for Disease Control 2019), burdening the economy with an estimated $\$ 11.2$ billion cost (Putri et al. 2018). Although efforts are ongoing to treat the virus, there still is no universal cure or preventative vaccine. This lack of treatment is due, in part, to the virus's ability to rapidly mutate and develop resistance (Hussain et al. 2017). Therefore, it is important to further understand how IAV and host cells interact during infection in order to develop new avenues for antiviral therapies.

The IAV genome is comprised of eight single-stranded, negative-sense RNA segments that are transcribed and replicated in the nucleus. Like many other nuclearexpressed viruses, several of the transcripts expressed by IAV undergo alternative splicing to generate distinct protein-coding open reading frames. Specifically, at least three of the eight RNA segments (M, NS, and PB2) have been reported to express at least two proteins through regulated splicing (Fig. 1; Palese and Shaw 2013; Yamayoshi et al. 2015; Fabozzi et al. 2018).

In the case of both mammalian and influenza genes, splicing occurs through recognition and joining of sequences in the RNA by the spliceosome-a multicomponent enzymatic complex (Fig. 2). Although the sequences specifically bound by the spliceosome are known as the "splice sites," sequences outside of these splice site regions bind regulatory factors to control the efficiency of spliceosomal binding and function (Fig. 2). Hundreds of RNA-binding proteins (RBPs) exist in mammalian cells that have been shown to be able to function as splicing regulatory factors (Fu and Ares 2014). Ultimately it is the activity of such regulatory factors that determine where and when splicing of any given transcript occurs. Such alternative splicing of mammalian genes often takes the form of skipping or inclusion of specific exons in a transcript. In contrast, the alternative splicing most observed among the IAV genes involves the retention or removal of a single intron (Fig. 1).

The IAV NS segment encodes the NS1 protein when the single intron in its transcript is retained, whereas the NS2 protein is encoded upon removal of the intron (Fig. 1). Both the NS1 and NS2 proteins are required for successful viral replication. NS1 has multiple functions in countering the antiviral response of host and promoting IAV gene expression, whereas NS2 promotes vRNA export and packaging and forms part of the viral particle (Palese and Shaw 2013). The first 56 nt of the NS1 and NS2 transcripts are identical, resulting in 13 shared aminoterminal amino acids. However, upon removal of the intron, the reading frame is altered and the downstream amino acids are divergent between NS1 and NS2. Similarly, recent work by Yamayoshi et al. show that the PB2 segment contains a putative intronic sequence from nucleotides 1513 to 1894 of the 2341-nt transcript (Fig. 1; Yamayoshi et al. 2015) that, when removed, encodes a protein termed PB2-S1. Interestingly, not all IAV strains express this splicing pattern and resultant protein, and PB2-S1 null viruses do not show altered viral replication rates. Therefore, the significance of PB2 segment splicing remains to be determined.

$\mathrm{M}$ segment splicing is perhaps the best characterized of all IAV segments at both the functional and mechanistic

(C) 2019 Thompson and Lynch. This article is distributed under the terms of the Creative Commons Attribution-NonCommercial License, which permits reuse and redistribution, except for commercial purposes, provided that the original author and source are credited. 


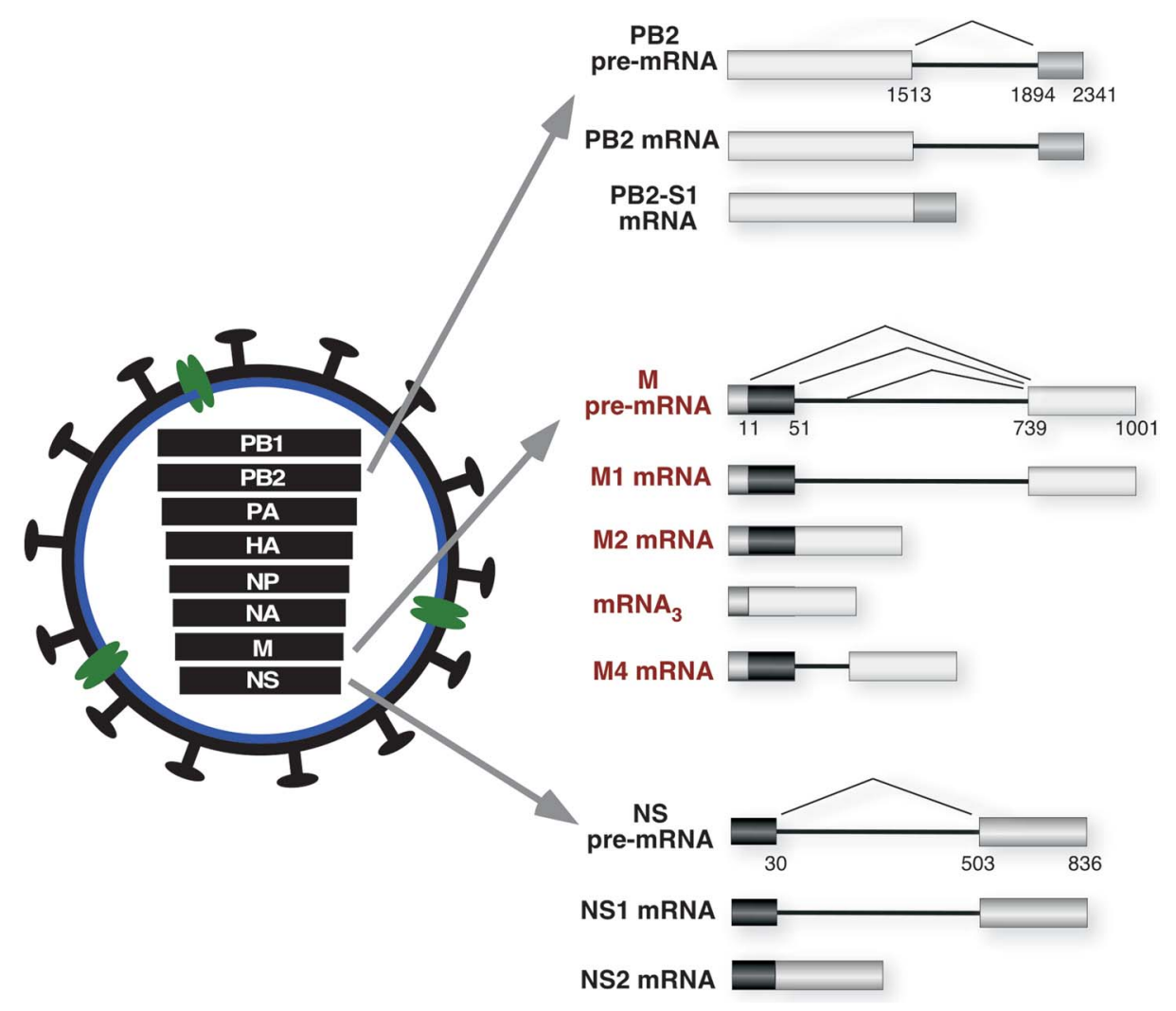

Figure 1. Diagram of influenza virus A showing the eight genome segments and the splicing patterns of the three segments best documented to undergo alternative splicing.

level. Like the other IAV segments described above, M segment splicing involves removal or retention of an intron. To date, the $\mathrm{M}$ segment has been shown to produce four alternate RNA isoforms: M1, M2, $\mathrm{mRNA}_{3}$, and M4 (Fig. 1). The M2, $\mathrm{mRNA}_{3}$, and M4 isoforms all share a common $3^{\prime}$ splice site ( $3^{\prime} \mathrm{SS}$ ) joined to different $5^{\prime} \mathrm{SSs}$, whereas the M1 isoform is the unspliced form. The M1 and M2 isoforms are the most abundant of the M segment
RNAs and are both translated into proteins that are necessary for the viral life cycle (Palese and Shaw 2013). In contrast, the function of $\mathrm{mRNA}_{3}$ and M4 splice isoforms is not understood. Jackson and Lamb showed that deletion of the $\mathrm{mRNA}_{3}$ splicing isoform does not influence IAV replication in cell culture (Jackson and Lamb 2008), whereas the M4 isoform exists in only trace amounts and has not been explored functionally.

A

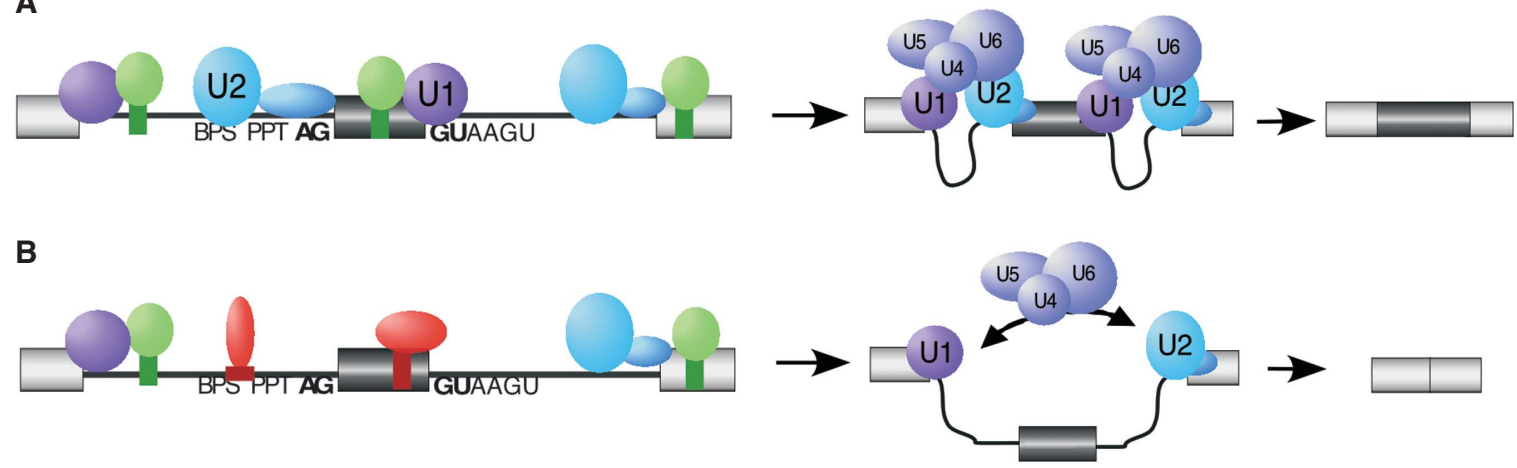

Figure 2. Diagram of the regulation of exon inclusion via RNA-binding proteins (RBPs). Boxes are exons; lines are introns. Conserved sequences at the splice sites are shown. Blue/purple circles labeled "U" are core spliceosome components that assemble in a step-wise fashion on the substrate. Green $(A)$ and red $(B)$ circles represent RBPs functioning to recruit the spliceosome to enhance exon inclusion $(A)$ or repress spliceosome recognition of splice sites to induce exon skipping $(B)$. 
At least for the NS1/NS2 and M1/M2 proteins, the balance of expression is critical for viral replication. Therefore, the splicing of the NS and M segment RNAs must be carefully regulated. The control of NS1 to NS2 splicing has yet to be fully understood. What is known is that NS1 to NS2 splicing is regulated by a weak $5^{\prime}$ splice site (5'SS) that diverges from the canonical sequence (Dubois et al. 2014). This deviation from the canonical motif results in altered base-pairing of the U1 snRNP to the 5' SS leading to inefficient splicing. Interestingly, Chua et al. propose that the NS1 5'SS is weak by design (Chua et al. 2013). In their model, they show that inefficient splicing causes NS2 levels to slowly accumulate throughout IAV infection at a rate that is advantageous to the virus. In contrast to NS, regulation of the $M$ segment splicing has been studied more thoroughly and occurs through regulation of both the $5^{\prime}$ and $3^{\prime}$ splice sites by the activity of host RBPs. Here we describe work by ourselves and others in defining the mechanism by which $\mathrm{M}$ segment splicing is regulated by host proteins, what this may mean for the splicing of host genes, and how the regulation of host and viral splicing regulation together impact viral replication.

\section{REGULATION OF IAV M SEGMENT SPLICING}

As mentioned above, all of the spliced forms of the M segment utilize a common 3'SS (Fig. 1). Previous work has shown that host protein SRSF1 can repress this 3'SS, thus promoting the production of the M1 isoform over the spliced variants (Shih and Krug 1996). In addition, structural analysis of the 3'SS has shown that 3'SS availability, and thus spliceosomal recognition of the intron-exon boundary, may be regulated via the confirmation of the 3'SS (Moss et al. 2012). These 3'SS studies provide an understanding of how high levels of the M1 isoform are produced; however, they do not address why M2 is the predominant spliced form. A previous model for $\mathrm{M}$ segment 5'SS selection suggested that $\mathrm{mRNA}_{3}$ splicing was repressed by the binding of viral polymerase components over the $\mathrm{mRNA}_{3} 5^{\prime} \mathrm{SS}$ to promote M1 and M2 splice products (Shih et al. 1995). However, a later study refuted this claim by showing that a IAV protein with RNA-binding activity, NS1, is sufficient to repress $\mathrm{mRNA}_{3}$ production and is likely a key contributor in the production of M1 and M2 splice products over $\mathrm{mRNA}_{3}$ (Robb and Fodor 2012). The mechanism by which NS1 controls M1 to M2 splicing was initially unclear. However, NS1 has a large interactome that includes many host RBPs (Thulasi Raman and Zhou 2016; Kuo et al. 2018), suggesting that host proteins might likewise contribute significantly to the regulation of M1 versus M2 expression.

One of the earliest characterized binding partners of the IAV NS1 protein is the aptly named NS1 binding protein (NS1-BP) (Wolff et al. 1998). NS1-BP localizes to nuclear speckles, a subnuclear compartment that harbors a high concentration of splicing-related proteins (Wolff et al. 1998). An important breakthrough in understanding the regulation of M2 splicing came with the demonstration that knockdown of NS1-BP results in decreased levels of
M2 and increased levels of M1, suggesting it had a direct role in M segment splicing regulation (Tsai et al. 2013). Additionally, the same study showed that knockdown of hnRNP K, a known host splicing regulator and binding partner of NS1-BP, also resulted in inhibition of M2 splicing (Tsai et al. 2013). Interestingly, NS1 has been proposed to cause relocalization of NS1-BP and other splicing factors away from nuclear speckles (Wolff et al. 1998; Fortes et al. 1995). To determine if subnuclear localization of proteins or RNA is important to the splicing of the M segment, a series of microscopy experiments were done to follow the localization of M1 and M2 splice isoforms in the context of NS1, NS1-BP, and hnRNP K depletion (Mor et al. 2016). Several key observations were made in this study. First, the authors show that M segment RNA localizes to nuclear speckles and that this localization is dependent on NS1 and NS1-BP. Second, M2 RNA is enriched specifically at speckles, suggesting that M1 to M2 splicing takes place within or in close proximity to nuclear speckles. Finally, although hnRNP K is not required for localization of M1 RNA to speckles, it is required to promote M2 splicing. Putting these observations together, the authors propose a model in which NS1, NS1-BP, and hnRNP K control M1 to M2 splicing through a nuclear speckle-dependent pathway (Fig. 3; Mor et al. 2016).

To dig more deeply into the mechanisms by which NS1$\mathrm{BP}$ and hnRNP K regulate M2 splicing, we recently carried out a biochemical study to localize the binding sites of NS1-BP and hnRNP K on the M1 transcript (Thompson et al. 2018). To identify the site(s) of hnRNP K and NS1$\mathrm{BP}$ binding to the M1 transcript we first carried out in vitro UV cross-linking assays with mammalian nuclear extract and a series of truncations of the M1 mRNA (Fig. 4A). These experiments showed that hnRNP K binds predominantly to a sequence within $30 \mathrm{nt}$ downstream from the M2 5'SS (Thompson et al. 2018). Previous work has shown that hnRNP K binds preferentially to polycytosine (pC) tracts (Thisted et al. 2001). Notably, mutation of two pC tracts at nt 69-71 and 78-84, respectively, abrogates hnRNP K cross-linking to the 1-106 fragment (Fig. 4A). The binding of hnRNP K close to the M2 5'SS, and the

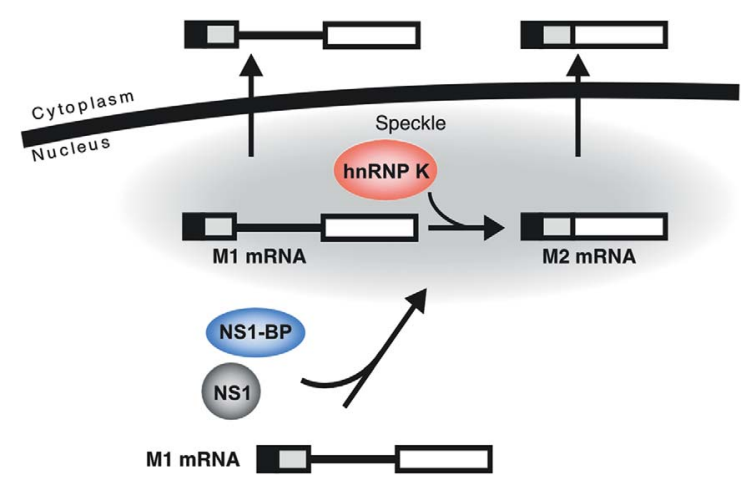

Figure 3. Diagram of the role of NS1, NS1-BP, and hnRNP K in promoting M2 splicing. NS1 and NS1-BP traffic the unspliced message to nuclear speckles. Within speckles hnRNP K promotes M1 to M2 splicing. Whether or not the message is spliced, trafficking to the speckles enhances export to the cytoplasm. 
A

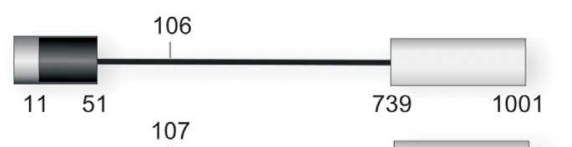

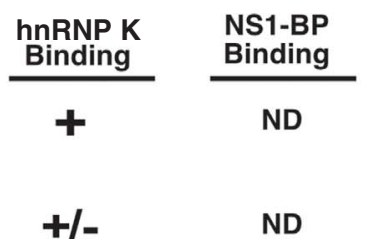

ND

$+$
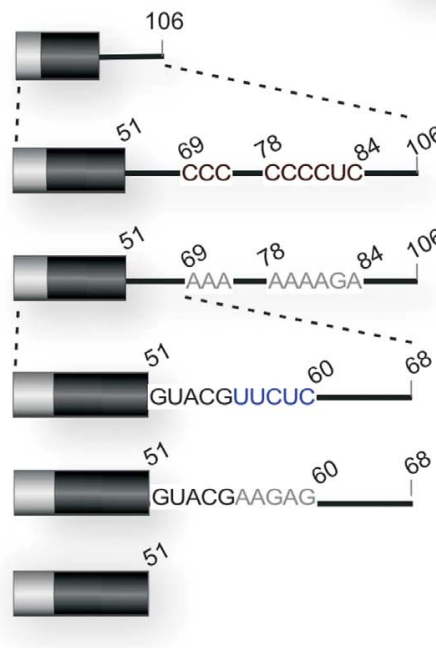

B
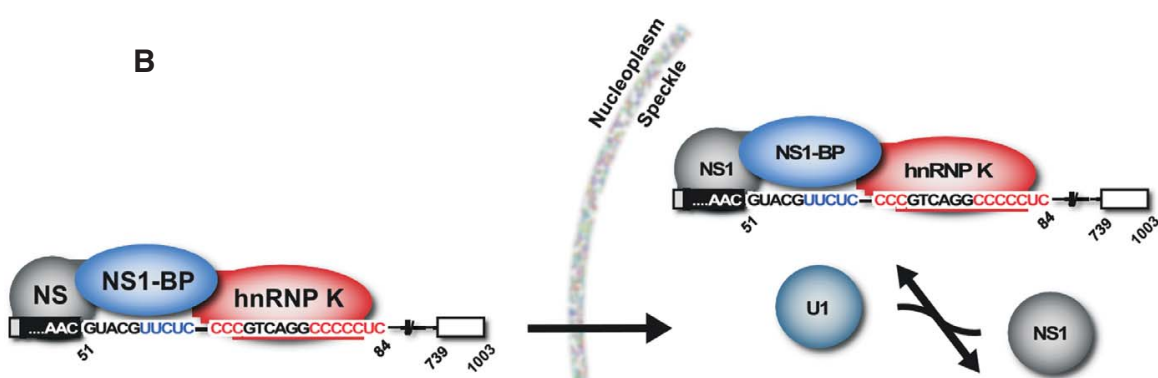

NS1

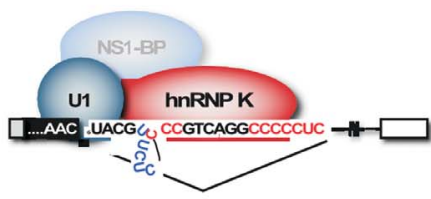

Figure 4. Sequences around the M2 5'SS are essential for recruitment of hnRNP K and NS1-BP. $(A)$ Schematics indicate substrates used in UV cross-linking assays. The presence or absence of association of hnRNP K and NS1-BP with each indicated substrate is indicated by + and - , respectively. (ND) Not determined. (B) Detailed model for the association of hnRNP K and NS1-BP with the M substrate to control splicing to M2. Mutant sequences are in gray; wild-type sequences are in maroon, blue, and black.

fact that this protein directly promotes M2 splicing, led us to ask if hnRNP K promotes the recruitment of the spliceosome to the 5'SS. Specifically, we investigated the impact of hnRNP K binding on the recruitment of the U1 snRNP - a complex of RNA (the U1 snRNA) and proteins that initially recognizes and binds 5'SS. By both RNA affinity and psoralen cross-linking assays, we indeed find that binding of the U1 snRNA and its associated proteins to the M2 5'SS is promoted in an hnRNP Kdependent manner. Together these data provide a clear model for how hnRNP $\mathrm{K}$ enhances the production of the M2 RNA in the speckles through facilitating the recruitment of the U1 snRNP (Fig. 4B).
Interestingly, a cross-linking signal was also observed for NS1-BP upon either mutation or deletion of the $\mathrm{pC}$ tracts (Fig. 4A). Further truncations of the M1 transcript revealed this apparent NS1-BP binding to be immediately overlapping the M2 5'SS sequence. However, as NS1-BP does not contain any canonical RNA-binding domains (Adams et al. 2000), we consider this interaction to be dependent on protein-protein interactions. Consistent with this conclusion, the presence of NS1, either in vitro or in infected cells, results in a different association of NS1-BP with the M transcript, in which the association of NS1-BP with RNA is dependent on the proximal binding of hnRNP $\mathrm{K}$ but not sequences within the 5'SS 
(Thompson et al. 2018). Because NS1-BP can interact in a trimeric complex with NS1 and hnRNP K, these data suggest that NS1 and hnRNP K effectively "hold" NS1BP over the 5'SS through protein-protein interactions. This model suggests that this trimeric complex thus hinders splicing until the M RNA is trafficked to the nuclear speckles, where we propose that the high level of splicing factors and the activity of hnRNP $\mathrm{K}$ favor recruitment of the U1 snRNP and the productive use of the M2 5'SS (Fig. 4B).

\section{REGULATION OF HOST SPLICING BY INFLUENZA INFECTION}

Although NS1-BP has been studied with respect to IAV and the viral NS1 protein, its role in uninfected cells has not been well-characterized. The observation that NS1-BP associates with RNA in the absence of virus led to the question of whether NS1-BP might have activity as a regulator of splicing of human genes. We therefore carried out a quantitative analysis of splicing of approximately 5500 known alternative exons in A549 cells in the absence or presence of knockdown of NS1-BP or hnRNP K using the previously described RASL platform (Martinez et al. 2015; Li et al. 2012). Consistent with the previously described role of hnRNP $\mathrm{K}$ as a splicing regulator, we observed that splicing of approximately 200 out of the approximately 5500 exons surveyed is dependent on hnRNP K (Fig. 5A). Strikingly, more than one-half of these hnRNP $\mathrm{K}$-dependent exons were also regulated in an NS1-BP-dependent manner, whereas only a handful of exons were identified as potentially NS1-BP-dependent, but not dependent on hnRNP K (Fig. 5A). Moreover, although NS1-BP and hnRNP K both showed enhancer and silencer activities (i.e., increased or decreased exon inclusion), almost all of the NS1-BP and hnRNP K-dependent exons were regulated in the same direction by both of these proteins (Fig. 5B), consistent with a model in which these proteins are working in concert. The lim- ited scope of splicing events interrogated by RASL makes it unfeasible to assess sequence enrichment within the hnRNP K and NS1-BP coregulated genes. However, several of the NS1-BP/hnRNP K-regulated host genes show obvious proximal $\mathrm{pY}$ and $\mathrm{pC}$ tracts downstream from the enhanced 5'SS, similar to the M2 5'SS (Thompson et al. 2018). Taken together, these data reveal a previously unappreciated function for NS1-BP in host gene regulation and suggest that hnRNP K and NS1-BP form a complex that specifically regulates a subset of host splicing events.

The fact that hnRNP K and NS1-BP coordinately regulate host splicing events also indicates that IAV hijacks a preexisting widespread cellular splicing regulatory relationship between hnRNP K and NS1-BP to carry out its own M1 to M2 splicing. Such a model immediately raises the question of whether IAV hijacking of hnRNP K and NS1-BP also perturbs host splicing through either sequestering these proteins away from host targets or redirecting their activity to other genes beyond the M segment. Indeed, changes in the splicing patterns of human genes upon IAV infection was recently reported by Fabozzi et al. (2018). Using seasonal and laboratory strains of IAV H3N2, these authors showed IAV-induced alternative splicing changes in at least 775 genes (Fabozzi et al. 2018), although this reported impact of IAV on splicing is likely an underestimate given the relatively low sequencing depth in the study.

To specifically ask if hnRNP K and NS1-BP coregulated host splicing events are altered in the context of IAV infection, we first directly assessed the splicing of several of the genes from the RASL study for changes upon infection with the WSN strain of IAV. Notably, we observe IAV-induced changes in $>60 \%$ of the genes tested (Thompson et al. 2018). Moreover, the impact of IAV infection on splicing implies two distinct mechanisms. For example, in the case of the genes CASP 8 and INF2, the impact of IAV infection was the opposite of that observed upon hnRNP K and NS1-BP knockdown, suggesting that IAV enhances the activity of hnRNP K and NS1-BP on some host target genes (Fig. 6). In contrast,
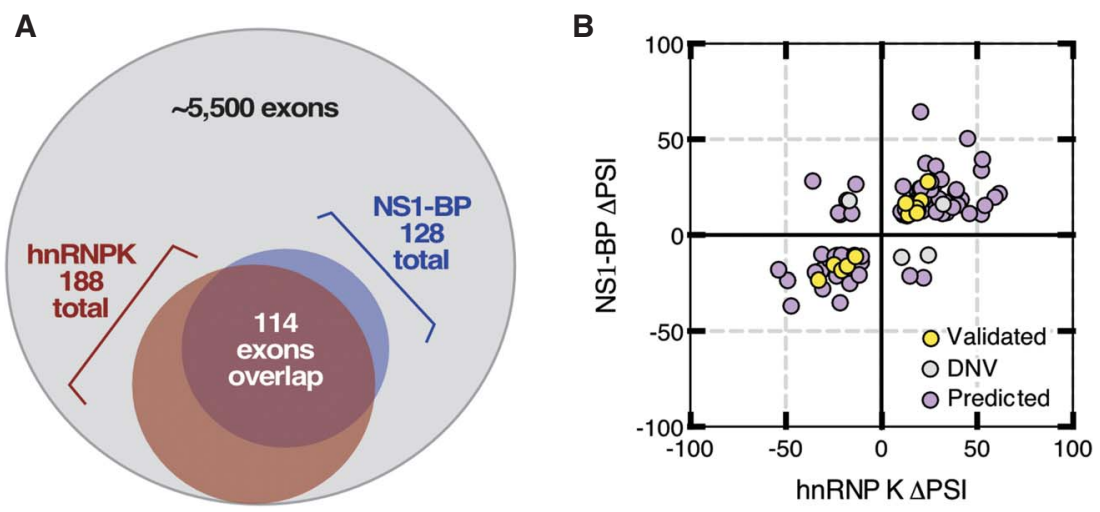

Figure 5. (A) Number of total surveyed exons that are differentially spliced upon depletion of hnRNP K (red), NS1-BP (blue), with overlap shown in purple. $(B)$ Correlation of the impact on exon inclusion of depletion of hnRNP K or NS1-BP. Purple are events from overlap in $A$. Yellow and gray represent those events validated or not, respectively, by reverse transcription polymerase chain reaction (RT-PCR). Plotted is the difference in percent spliced isoform ( $\triangle \mathrm{PSI})$ between wild type and NS1-BP $(y$-axis) or hnRNP K ( $x$-axis) depletion. 

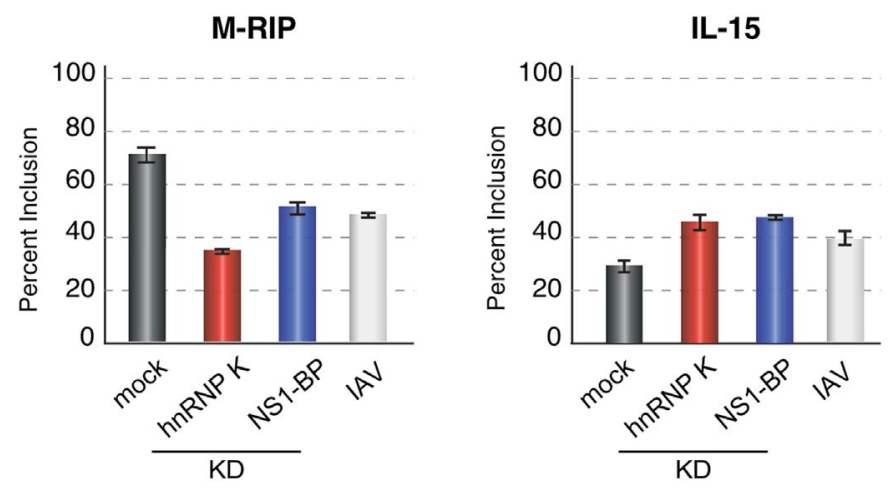

CASP8

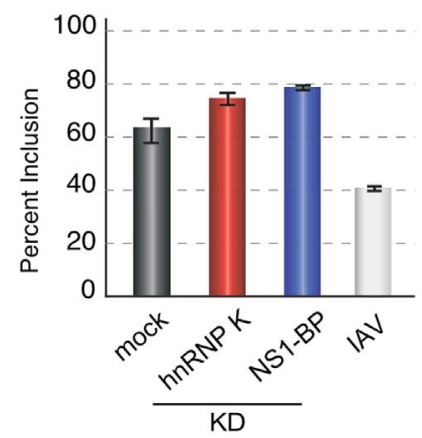

INF2

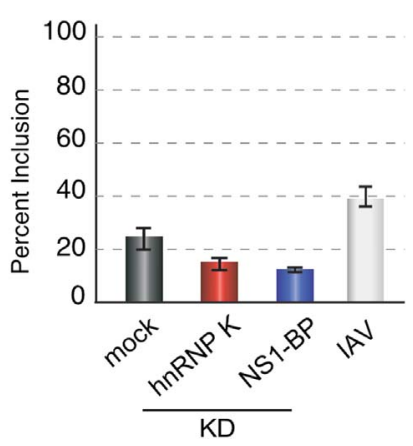

Figure 6. Examples of genes that are differentially spliced upon depletion of hnRNP K and NS1-BP and are also regulated upon IAV infection. The percent of transcripts including the variable exon are plotted for each condition. (KD) The protein has been targeted for knockdown.

splicing of $M-R I P$ and $I L-15$ in IAV-infected cells phenocopied hnRNP K or NS1-BP depletion (Fig. 6), suggesting that for a different set of target genes IAV infection inhibits hnRNP K/NS1-BP association and/or function.

To expand this analysis, we have recently carried out a high-depth RNA-seq experiment comparing IAV-infected versus uninfected A549 cells. Specifically, we sequenced poly(A) RNA from cells 6 and $12 \mathrm{~h}$ after infection with the WSN strain of IAV and compared the splicing to that of poly(A) RNA from uninfected cells using the MAJIQ algorithm (Vaquero-Garcia et al. 2016). The robustness of infection was confirmed by both the presence of reads from IAV RNA as well as up-regulation of several interferon-inducible genes. Strikingly, we observe approximately 900 significant splicing changes in host transcripts at both time points of IAV infection, impacting approximately 600 genes. The vast majority $(\sim 90 \%)$ of these genes do not show any changes in expression levels in response to IAV, indicating that regulation of these transcripts is solely at the level of splicing. Over the time course of the infection, these splicing events tend to increase their difference relative to wild type, consistent with what would be expected for IAV-induced splicing regulation.

Importantly, several of the IAV-induced splicing changes occur in genes that have previously been described to regulate IAV infection (Fig. 7). For example, CLK1 is known to be an upstream regulator of splicing of host and viral transcripts through interactions with other host splicing factors and is particularly important for the production of IAV M2 (Duncan et al. 1997; Aubol et al. 2016; Dominguez et al. 2016). IAV-induced splicing of CLK1 is predicted to increase the production of the full-length protein by promoting inclusion of all exons (Fig. 7). Therefore IAV-regulation of $C L K 1$ via splicing is likely to promote viral replication through promoting a correct balance of M1 to M2 expression. Similarly, RAB11FIP3 and PPIP5K2, two additional genes harboring IAVinduced splicing events, have been shown to regulate IAV replication (Fig. 7). RAB11FIP3 competes with IAV vRNPs for RAB11 on endosomes in the particle assembly pathway, thus regulating viral egress (Vale-Costa et al. 2016), whereas PPIP5K2 is required for efficient IFN- $\beta$ production (Pulloor et al. 2014), thus inhibiting viral replication via the innate immune response. The IAV-induced splicing changes in both of these genes results in the insertion of additional peptide sequences within the canonical encoded protein. The functional impact of these altered reading frames remains to be explored and will be an interesting area of future study (see Conclusion).

\section{CONCLUSION}

The work described above highlights how IAV uses host machinery to expand the coding potential of its genome 


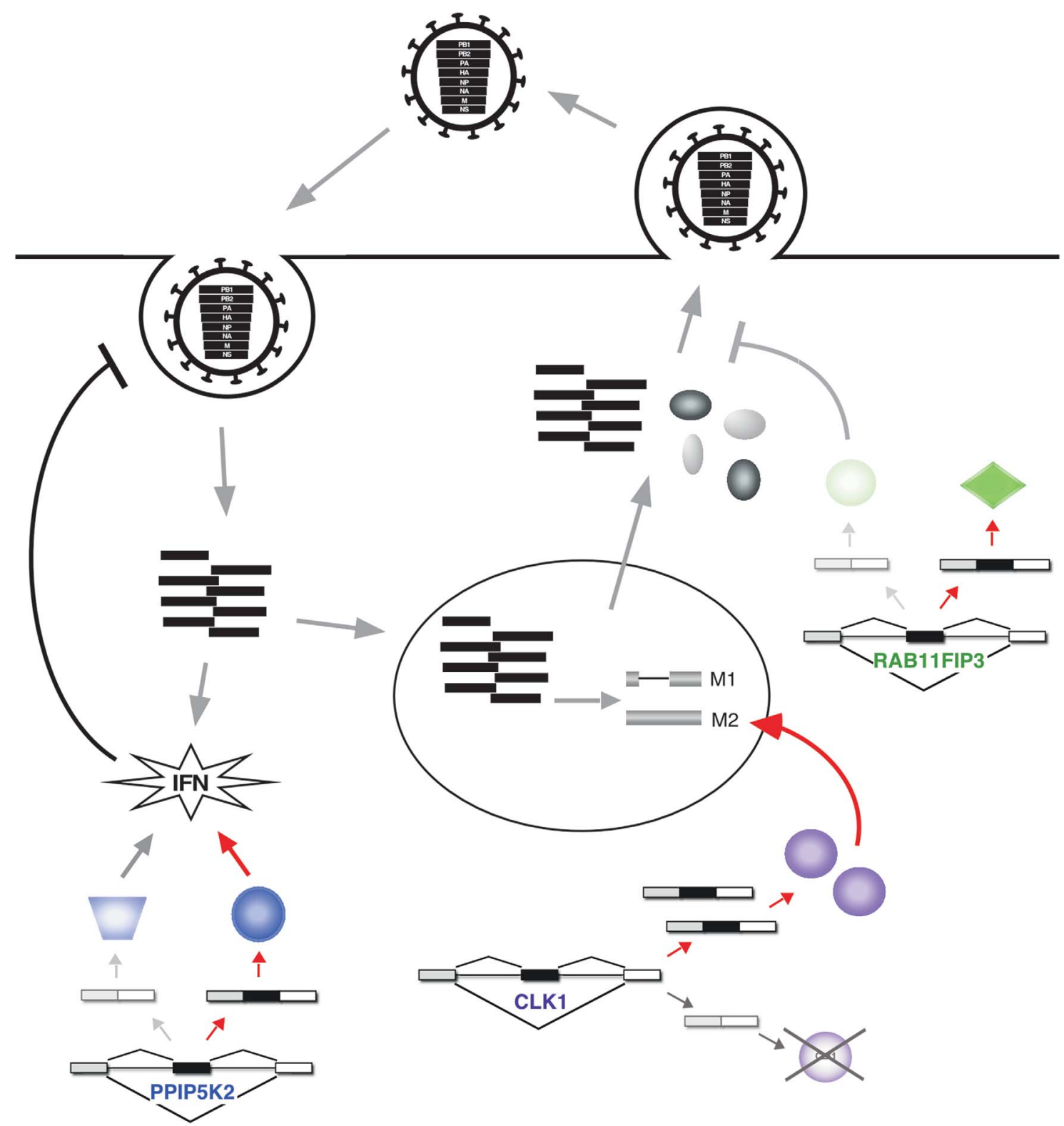

Figure 7. Model of the putative role of CLK1, RAB11FIP3, and PPIP5K2 in IAV replication and the possible impact of alternative splicing of the genes encoding these proteins in promoting or limiting IAV infection. Gray arrows indicate normal viral life cycle; red arrows indicate viral-induced splicing patterns of the three genes and resulting predicted protein and functional impact.

through alternative splicing. In particular the splicing event we focus on, that of M1 to M2 splicing, is essential for viral replication. A major hurdle for the development of antiviral therapies is the ability of the virus to mutate and evolve rapidly. However, virus cannot confer the same adaptive response on host proteins. Therefore, identifying essential interactions between the host splicing regulatory machinery and IAV RNAs and proteins opens the door to therapeutic approaches that target host proteins to prevent viral replication.

Beyond the potential for novel therapeutics, the study of viral splicing provides important insights to general mechanisms of splicing regulation. For example, the require- ment for M1 RNA to be trafficked to nuclear speckles for subsequent export or splicing is unusual. Typically host genes are thought to be spliced in a cotranscriptional manner, with the splicing machinery being recruited from the speckles to the sites of transcription (Merkhofer et al. 2014). The fact that hnRNP K and NS1-BP regulate splicing of many host genes in addition to the $\mathrm{M}$ transcript suggests that a subset of host RNA may also be spliced in a speckle-dependent manner at least in the context of IAV infection. Indeed, the involvement of NS1 in trafficking and splicing the M segment RNA with hnRNP $\mathrm{K}$ and NS1-BP raises the possibility that NS1 could be directing a specific subset of hnRNP K/NS1-BP-regulated RNAs 
through nuclear speckles, which would otherwise be spliced cotranscriptionally. Recent experiments from the Fontoura laboratory do show that NS1 binds a population of host transcripts during IAV infection (Zhang et al. 2018). Therefore, determining if hnRNP K- and NS1BP-regulated splicing events are bound by NS1 could reveal host RNAs that might be subject to $\mathrm{M}$ segmentlike splicing mechanisms. Importantly, if it is determined that nuclear speckle-dependent splicing pathways are exclusively an IAV-driven phenomena, then targeting this pathway therapeutically may prove to be an additional viable antiviral strategy.

More broadly, a major open question is to define the mechanism(s) by which IAV infection alters host splicing. As described above, one potential mechanism is via IAVinduced changes in hnRNP K/NS1-BP activity. For example, the presence of NS1 could draw hnRNP K/NS1-BP to speckles, as discussed in the preceding paragraph, or could alter the recruitment of these host proteins to specific RNAs through a combination of protein-protein and protein-RNA interactions. Alternatively, the abundance of IAV M RNA could function as a molecular sponge to sequester hnRNP K/NS1-BP away from host transcripts, as has been shown to occur for other viral RNAs and host proteins (Barnhart et al. 2013; Michalski et al. 2019) Consistent with this model, our and other RNA-seq experiments have shown that during infection IAV RNA accounts for $>30 \%$ of polyadenylated transcripts in the cell.

Finally, the fact that IAV infection induces so many alterations in host splicing begs the question of whether these are advantageous for the virus, part of the host defense, or both. The fact that several genes that harbor IAV-regulated splicing events are known to have pro or antiviral activities strongly suggests that changes in host splicing induced upon viral infection impact viral replication. We predict that splicing changes induced specifically by the virus (e.g., via NS1 or viral RNA load) are likely to be proviral, as the virus would avoid causing such changes if they hinder viral replication. In contrast, it is possible that a subset of the observed splicing changes induced upon infection are actually part of the hostdriven innate immune response. Such innate immune triggered splicing has been observed in other systems (Carpenter et al. 2014) and would be expected to be antiviral. Future studies are needed to tease apart viral- versus host-driven splicing regulation and to specifically test the influence of alternative splicing on viral growth by specifically modulating splicing by antisense or CRISPR approaches.

\section{ACKNOWLEDGMENTS}

The authors thank our collaborators Beatriz Fontoura, Adolfo Garcia-Sastre, YuhMin Chook, and Sara Cherry for discussions throughout this work. K.W.L. is supported by National Institutes of Health (NIH) grant R35 GM118048, and M.G.T., K.W.L., and the work described herein are supported by NIH grant R01 AI125524.

\section{REFERENCES}

Adams J, Kelso R, Cooley L. 2000. The kelch repeat superfamily of proteins: propellers of cell function. Trends Cell Biol 10: 17-24. doi:10.1016/S0962-8924(99)01673-6

Aubol BE, Wu G, Keshwani MM, Movassat M, Fattet L, Hertel KJ, Fu X-D, Adams JA. 2016. Release of SR proteins from CLK1 by SRPK1: a symbiotic kinase system for phosphorylation control of pre-mRNA splicing. Mol Cell 63: 218-228. doi:10.1016/j.molcel.2016.05.034

Barnhart MD, Moon SL, Emch AW, Wilusz CJ, Wilusz J. 2013. Changes in cellular mRNA stability, splicing, and polyadenylation through HuR protein sequestration by a cytoplasmic RNA virus. Cell Rep 5: 909-917. doi:10.1016/j.celrep.2013 10.012

Carpenter S, Ricci EP, Mercier BC, Moore MJ, Fitzgerald KA. 2014. Post-transcriptional regulation of gene expression in innate immunity. Nat Rev Immunol 14: 361-376. doi:10.1038/ nri3682

Centers for Disease Control. 2019. Estimated influenza illnesses, medical visits, hospitalizations, and deaths averted by vaccination in the United States CDC. CDC, Atlanta.

Chua MA, Schmid S, Perez JT, Langlois RA, Tenoever BR. 2013. Influenza Avirus utilizes suboptimal splicing to coordinate the timing of infection. Cell Rep 3: 23-29. doi:10.1016/j.celrep .2012.12.010

Dominguez D, Tsai Y-H, Weatheritt R, Wang Y, Blencowe BJ, Wang Z. 2016. An extensive program of periodic alternative splicing linked to cell cycle progression. Elife 5: e10288. doi:10.7554/eLife.10288

Dubois J, Terrier O, Rosa-Calatrava M, De Virologie L, Virpath H, Vircell G, Claude U, Lyon B, De Lyon U. 2014. Influenza viruses and mRNA splicing : doing more with less. mBio 5: 113. doi:10.1128/mBio.00070-14

Duncan PI, Stojdl DF, Marius RM, Bell JC. 1997. In vivo regulation of alternative pre-mRNA splicing by the Clk1 protein kinase. Mol Cell Biol 17: 5996-6001. doi:10.1128/MCB.17 .10 .5996

Fabozzi G, Oler AJ, Liu P, Chen Y, Mindaye S, Dolan MA, Kenney H, Gucek M, Zhu J, Rabin RL, et al. 2018. Strandspecific dual RNA-seq of bronchial epithelial cells infected with influenza $\mathrm{A} / \mathrm{H} 3 \mathrm{~N} 2$ viruses reveals splicing of gene segment 6 and novel host-virus interactions. J Virol 92: e0051818. doi:10.1128/JVI.00518-18

Fortes P, Lamond A, Ortín J. 1995. Influenza virus NS1 protein alters the subnuclear localization of cellular splicing components. J Gen Virol 76: 1001-1007. doi:10.1099/0022-131776-4-1001

Fu X-D, Ares M. 2014. Context-dependent control of alternative splicing by RNA-binding proteins. Nat Rev Genet 15: 689701. doi: $10.1038 / \operatorname{nrg} 3778$

Hussain M, Galvin H, Haw TY, Nutsford A, Husain M. 2017. Drug resistance in influenza A virus: the epidemiology and management. Infect Drug Resist 10: 121-134. doi:10.2147/ IDR.S105473

Jackson D, Lamb RA. 2008. The influenza A virus spliced messenger RNA M mRNA3 is not required for viral replication in tissue culture. J Gen Virol 89: 3097-3101. doi:10.1099/vir.0 .2008/004739-0

Kuo R-L, Chen C-J, Tam E-H, Huang C-G, Li L-H, Li Z-H, Su PC, Liu H-P, Wu C-C. 2018. Interactome analysis of NS1 protein encoded by influenza A H7N9 virus reveals an inhibitory role of NS1 in host mRNA maturation. J Proteome Res 17: 1474-1484. doi:10.1021/acs.jproteome.7b00815

Li H, Qiu J, Fu XD. 2012. RASL-seq for massively parallel and quantitative analysis of gene expression. Curr Protoc Mol Biol Chapter 4: Unit 4.13.1-9. doi:10.1002/0471142727 .mb0413s 98

Martinez NM, Agosto L, Qiu J, Mallory MJ, Gazzara MR, Barash Y, Fu X-D, Lynch KW. 2015. Widespread JNK-dependent alternative splicing induces a positive feedback loop through CELF2-mediated regulation of MKK7 during T-cell activation. Genes Dev 29: 2054-2066. doi:10.1101/gad.267245.115 
Merkhofer EC, Hu P, Johnson TL. 2014. Introduction to cotranscriptional RNA splicing. Methods Mol Biol 1126: 83-96. doi:10.1007/978-1-62703-980-2 6

Michalski D, Ontiveros JG, Russo J, Charley PA, Anderson JR, Heck AM, Geiss BJ, Wilusz J. 2019. Zika virus noncoding sfRNAs sequester multiple host-derived RNA-binding proteins and modulate mRNA decay and splicing during infection. J Biol Chem 294: 16282-16296. doi:10.1074/jbc.RA119 .009129

Mor A, White A, Zhang K, Thompson M, Esparza M, MuñozMoreno R, Koide K, Lynch KW, García-Sastre A, Fontoura BMA. 2016. Influenza virus mRNA trafficking through host nuclear speckles. Nat Microbiol 1: 16069. doi:10.1038/nmicro biol.2016.69

Moss WN, Dela-Moss LI, Kierzek E, Kierzek R, Priore SF, Turner DH. 2012. The 3' splice site of influenza A segment 7 mRNA can exist in two conformations: a pseudoknot and a hairpin. PLOS ONE 7: e38323. doi:10.1371/journal.pone .0038323

Palese P, Shaw M. 2013. Orthomyxoviridae. In Fields virology, 6th ed. (ed. Fields BN, Knipe DM, Howley PM), pp. 11511185. Lippincott Williams \& Wilkins, Philadelphia.

Pulloor NK, Nair S, Kostic AD, Bist P, Weaver JD, Riley AM, Tyagi R, Uchil PD, York JD, Snyder SH, et al. 2014. Human genome-wide RNAi screen identifies an essential role for inositol pyrophosphates in type-I interferon response. PLoS Pathog 10: e1003981. doi:10.1371/journal.ppat.1003981

Putri WCWS, Muscatello DJ, Stockwell MS, Newall AT. 2018. Economic burden of seasonal influenza in the United States. Vaccine 36: 3960-3966. doi:10.1016/j.vaccine.2018 .05 .057

Robb NC, Fodor E. 2012. The accumulation of influenza A virus segment 7 spliced mRNAs is regulated by the NS1 protein. $J$ Gen Virol 93: 113-118. doi:10.1099/vir.0.035485-0

Shih SR, Krug RM. 1996. Novel exploitation of a nuclear function by influenza virus: the cellular SF2/ASF splicing factor controls the amount of the essential viral M2 ion channel protein in infected cells. EMBO $J$ 15: 5415-5427. doi:10 $.1002 / \mathrm{j} .1460-2075.1996 . t b 00925 . x$

Shih SR, Nemeroff ME, Krug RM. 1995. The choice of alternative $5^{\prime}$ splice sites in influenza virus M1 mRNA is regulated by the viral polymerase complex. Proc Natl Acad Sci 92: 6324 6328. doi:10.1073/pnas.92.14.6324
Thisted T, Lyakhov DL, Liebhaber SA. 2001. Optimized RNA targets of two closely related triple KH domain proteins, heterogeneous nuclear ribonucleoprotein $\mathrm{K}$ and $\alpha \mathrm{CP}-2 \mathrm{KL}$, suggest distinct modes of RNA recognition. J Biol Chem 276: 17484-17496. doi:10.1074/jbc.M010594200

Thompson MG, Muñoz-Moreno R, Bhat P, Roytenberg R, Lindberg J, Gazzara MR, Mallory MJ, Zhang K, García-Sastre A, Fontoura BMA, et al. 2018. Co-regulatory activity of hnRNP $\mathrm{K}$ and NS1-BP in influenza and human mRNA splicing. Nat Commun 9: 2407. doi:10.1038/s41467-018-04779-4

Thulasi Raman SN, Zhou Y. 2016. Networks of host factors that interact with NS1 protein of influenza Avirus. Front Microbiol 7: 654. doi:10.3389/fmicb.2016.00654

Tsai P-L, Chiou N-T, Kuss S, García-Sastre A, Lynch KW, Fontoura BMA, Garcia-Sastre A, Lynch KW, Fontoura BMA. 2013. Cellular RNA binding proteins NS1-BP and hnRNP K regulate influenza A virus RNA splicing. PLoS Pathog 9: e1003460. doi:10.1371/journal.ppat.1003460

Vale-Costa S, Alenquer M, Sousa AL, Kellen B, Ramalho J, Tranfield EM, Amorim MJ. 2016. Influenza A virus ribonucleoproteins modulate host recycling by competing with Rab11 effectors. J Cell Sci 129: 1697-1710. doi:10.1242/jcs .188409

Vaquero-Garcia J, Barrera A, Gazzara MR, González-Vallinas J, Lahens NF, Hogenesch JB, Lynch KW, Barash Y. 2016. A new view of transcriptome complexity and regulation through the lens of local splicing variations. Elife 5: e11752. doi:10.7554/ eLife. 11752

Wolff T, O’Neill RE, Palese P. 1998. NS1-binding protein (NS1$\mathrm{BP})$ : a novel human protein that interacts with the influenza A virus nonstructural NS1 protein is relocalized in the nuclei of infected cells. J Virol 72: 7170-7180.

World Health Organization. 2019. WHO influenza (seasonal). WHO, Geneva.

Yamayoshi S, Watanabe M, Goto H, Kawaoka Y. 2015. Identification of a novel viral protein expressed from the PB2 segment of influenza A virus. J Virol 90: 444-456. doi:10.1128/JVI .02175-15

Zhang L, Wang J, Muñoz-Moreno R, Kim M, Sakthivel R, Mo W, Shao D, Anantharaman A, García-Sastre A, Conrad NK, et al. 2018. Influenza virus NS1 protein-RNA interactome reveals intron targeting. J Virol 92: e01634-18. doi:10.1128/ JVI.01634-18 


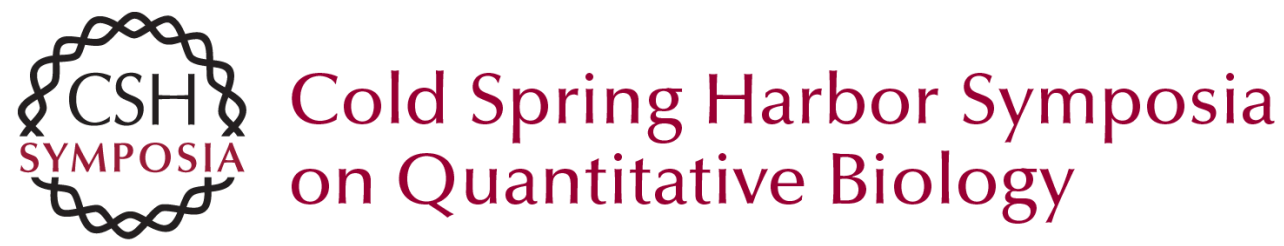

\section{Functional and Mechanistic Interplay of Host and Viral Alternative Splicing Regulation during Influenza Infection}

Matthew G. Thompson and Kristen W. Lynch

Cold Spring Harb Symp Quant Biol 2019 84: 123-131

Access the most recent version at doi:10.1101/sqb.2019.84.039040

References This article cites 34 articles, 10 of which can be accessed free at: http://symposium.cshlp.org/content/84/123.full.html\#ref-list-1

Creative This article is distributed under the terms of the

Commons http://creativecommons.org/licenses/by-nc/4.0/, which permits reuse and License redistribution, except for commercial purposes, provided that the original author and source are credited.

Email Alerting Receive free email alerts when new articles cite this article - sign up in Service the box at the top right corner of the article or click here. 